\begin{tabular}{|c|c|}
\hline Title & W avefront superposition method for accurate and efficient mode conversion \\
\hline Author(s) & Maeda, Tomohiro; Okamoto, A tsushi; Ogawa, Kazuhisa; Tomita, A kihisa \\
\hline Citation & $\begin{array}{l}\text { A pplied optics, 58(25), } 6899-6905 \\
\text { https://doi.org/10.1364/A 0.58.006899 }\end{array}$ \\
\hline Issue Date & 2019-09-01 \\
\hline Doc URL & http:/hdl. handle.net/2115/79207 \\
\hline Rights & $\begin{array}{l}\text { ( } 2019 \text { Optical Society of A merica. One print or electronic copy may be made for personal use only. Systematic } \\
\text { reproduction and distribution, duplication of any material in this paper for a fee or for commercial purposes, or } \\
\text { modifications of the content of this paper are prohibited. }\end{array}$ \\
\hline Type & article (author version) \\
\hline File Information & ao-58-25-6899_for_HUSCAP.pdf \\
\hline
\end{tabular}

Instructions for use 


\title{
Wavefront superposition method for accurate and efficient mode conversion
}

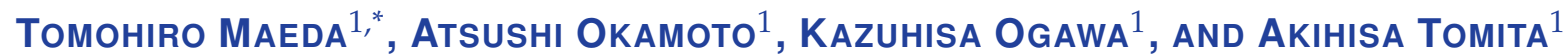 \\ ${ }^{1}$ Graduate School of Information Science and Technology, Hokkaido University, Sapporo, 060-0814, Japan \\ *Corresponding author: maeda@optnet.ist.hokudai.ac.jp \\ Compiled October 25, 2019
}

A wavefront superposition (WS) method is proposed for accurate and efficient mode conversion in modedivision multiplexing transmission. The WS method converts an input beam to the WS state, which is composed of the conversion target and radiation modes of a few-mode fiber. The appropriate weighting for the modal component of the WS state enables more efficient conversion than the conventional method in which the output beam consists only of the conversion target. Further, since the components of the radiation modes in the output are eliminated by the mode-filtering property of the few-mode fiber, no modal crosstalk occurs in the WS method. We examine the conversion performance of the WS method by a numerical simulation for the mode-multiplexing numbers $3,6,10$, and 15. The WS method shows a 2.4 dB higher efficiency than the conventional method, while maintaining an extremely low modal crosstalk (less than $-80 \mathrm{~dB}$ ) even when the number of multiplexed modes is 15. () 2019 Optical Society of America

http://dx.doi.org/10.1364/ao.XX.XXXXXX

\section{INTRODUCTION}

In recent decades, the technologies related to optical fiber communication have developed remarkably and have supported a fast developing information society. However, network traffic has increased drastically due to the increase in the number of internet users and data content, and it is approaching the theoretical limit of the transmission capacity of the current optical communication system based on single-mode fibers (SMFs) [13]. Since this trend of increasing network traffic is expected to continue in future [4], it is necessary to overcome the capacity limit in order to support the future demands of the network capacity. Space-division multiplexing (SDM) has been intensively studied to overcome the capacity limit of the current optical transmission system [5-12]. To implement SDM, there are two approaches: core multiplexing using multi-core fibers [13, 14] and mode-division multiplexing (MDM) using few- or multimode fibers (FMFs or MMFs) [15-18]. Since these multiplexing techniques can be used in combination with the current multiplexing techniques (e.g., wavelength-division multiplexing), it is expected to enhance the transmission capacity in proportion to the number of cores or propagation modes in the fiber. Soma et al. performed a transmission experiment at over $10 \mathrm{~Pb} / \mathrm{s}$ capacity by combining core multiplexing and MDM [11].

A mode converter, which converts a fundamental mode of an SMF to a propagation mode of an FMF, is one of the essential components to realize an MDM transmission system. The parameters representing the performance of a mode converter are its accuracy and efficiency that are expressed, respectively, by the modal crosstalk (MXT) and the optical loss occurring in the mode conversion. The efficiency is obviously an important parameter that determines the transmission cost. On the other hand, the accuracy of a mode converter is not serious depending on the transmission system, since the MXT can be compensated by multiple-input multiple-output (MIMO) digital signal processing (DSP). However, the increase in mode-multiplexing number to increase transmission capacity enhances the MIMO complexity and makes real-time processing difficult. To reduce the MIMO complexity, weakly-coupled MDM with partial MIMO, which is applied only to degenerate modes (e.g., $\mathrm{LP}_{11 \mathrm{e}}$ and $\left.\mathrm{LP}_{11 \mathrm{o}}\right)$, has attracted the attention of researchers $[15,19,20]$. In weakly-coupled MDM transmission, the accuracy of the mode converter used in the mode multi/demultiplexer is important because the MXT between modes that are not degenerate, such as $\mathrm{LP}_{01}$ and $\mathrm{LP}_{11 \mathrm{e}}$, cannot be compensated.

For mode conversion, a method based on phase-only modulation by a phase plate has been proposed [21, 22]. In this method, only the phase distribution of an input beam is modulated, and the intensity distribution is not changed. When the number of propagation modes in an FMF is small, this method is effective because the spatial orthogonality between converted beams is ensured only by phase modulation. In addition, high resolution is not required for the phase plate because a relatively low-order mode has a coarse phase distribution consisting of 0 and $\pi$. However, it is difficult to ensure the spatial orthogonality between beams modulated only by the phase distribution when the multiplexing number is large. Methods based on complex 
amplitude modulation have been proposed for accurate mode conversion [23-27]. Since these methods modulate not only the phase distribution but also the intensity distribution, the output beam excites only the target mode and no MXT occurs ideally. However, in these methods, the optical loss caused by converting the intensity distribution from the input Gaussian to the target mode cannot be avoided. Therefore, it can be said that a trade-off between accuracy and efficiency arises in conventional mode conversion methods.

In this paper, we propose an approach to achieve mode conversion with high accuracy and high efficiency by utilizing the mode filtering property of an FMF. In our method, referred to as wavefront superposition (WS) method, the output beam of a complex amplitude modulator is composed of the conversion target and radiation modes. Here, by determining the appropriate weighting for these modes, the output beam can contain a higher proportion of the target component than that in the conventional method, in which the output beam consists only of the conversion target. Moreover, since the components other than the conversion target are radiation modes, they radiate from the fiber through the propagation in the fiber and do not lead to MXT ideally. After evaluating the dependence on the input beam diameter, the conversion performance is compared under the optimal conditions for the proposed and conventional methods by a numerical simulation. To examine the applicability of the WS method to a large-scale MDM transmission system, the conversion performance is evaluated for the cases where the number of multiplexed modes are 3, 6, 10, and 15 .

\section{MODE CONVERSION AND MODE EXCITATION IN A FEW-MODE FIBER}

\section{A. Property of spatial light modulation}

Spatial light modulation is generally realized by a static optical mask or a spatial light modulator (SLM). The complex amplitude distribution of the modulated beam $E_{\text {out }}(x, y)$ is expressed as follows, using a function $f(x, y)$ representing the modulation introduced by the modulator.

$$
E_{\text {out }}(x, y)=f(x, y) \cdot E_{\text {in }}(x, y),
$$

where $E_{\mathrm{in}}(x, y)$ is the complex amplitude distribution of the input beam irradiating the modulator. In the mode conversion, the input beam is a Gaussian emerging from an SMF or a laser source. In the mode conversion based on phase-only modulation, the modulation function $f_{\mathrm{p}}(x, y)$ is expressed as

$$
f_{\mathrm{p}}(x, y)=\exp \left[i \phi_{\mathrm{t}}(x, y)\right]
$$

where $\phi_{\mathrm{t}}(x, y)$ is the phase distribution of the conversion target $E_{\mathrm{t}}(x, y)$. Here, since the phase distribution of the relatively loworder mode has a coarse distribution consisting of 0 and $\pi$, a phase plate created by the photolithographic process is often used as the modulator.

On the other hand, in the mode conversion based on complex amplitude modulation in which the output beam consists of only the conversion target mode, the modulation function $f_{\mathrm{c}}(x, y)$ is expressed as

$$
f_{\mathrm{c}}(x, y)=\left|\frac{E_{\mathrm{t}}(x, y)}{E_{\mathrm{in}}(x, y)}\right|\left[\max _{x, y}\left(\left|\frac{E_{\mathrm{t}}(x, y)}{E_{\mathrm{in}}(x, y)}\right|\right)\right]^{-1} \exp \left[i \phi_{\mathrm{t}}(x, y)\right] .
$$

Since the intensity modulation is performed only by spatial attenuation (but no amplification), it is necessary to normalize the output amplitude so as not to exceed the input on the entire modulation plane as expressed in the maximum term in Eq. Eq. (3). Therefore, a large optical loss cannot be avoided while obtaining the complete intensity distribution of the target mode from the input Gaussian beam, as shown in Fig. 1.

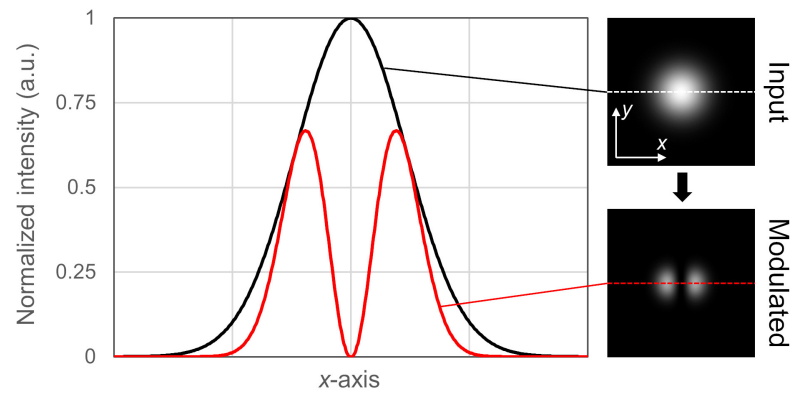

Fig. 1. Relationship between an input and output intensity profile obtained by intensity modulation in the mode conversion from a fundamental mode to $\mathrm{LP}_{110}$.

\section{B. Mode excitation in a few-mode fiber and the mode-filtering property}

As shown in Fig. 2, a beam coupled into an FMF excites plural modes. When the FMF has $N$ propagation modes, the output beam from a mode converter $E_{\text {out }}(x, y)$ is mathematically expressed as a linear combination of modes as follows:

$$
E_{\text {out }}(x, y)=\sum_{i=1}^{N} C_{i} P_{i}(x, y)+\sum_{i=N+1}^{\infty} C_{i} R_{i}(x, y)
$$

where $C_{i}$ is the coupling coefficient to the $i$-th mode. $P_{i}(x, y)$ and $R_{i}(x, y)$ are the complex amplitude distributions of the $i$-th propagation and radiation modes, respectively. The coupling efficiency $\eta_{i}=\left|C_{i}\right|^{2}$ represents the energy transition from the converted beam to the $i$-th mode. Here, since the energy coupled to the radiation modes is removed through the fiber propagation, it can be said that the FMF acts as a mode filter. If the complex amplitude distribution of the modulated beam completely accords with that of the target mode, only the target mode is excited. However, since phase-only modulation maintains the intensity distribution of the input beam, the converted beam may excite not only the target mode but also other propagation modes. The components of propagation modes other than the conversion target causes MXT in the MDM transmission system. In phase-only modulation, it is difficult to avoid MXT to the modes with the following characteristics.

- Modes having the same radial mode number with the conversion target, e.g., $\mathrm{LP}_{02}$ and $\mathrm{LP}_{03}$, when targeting $\mathrm{LP}_{01}$.

- Modes having an odd azimuthal mode number and having the same symmetry axis with the conversion target, e.g., $\mathrm{LP}_{31 \mathrm{e}}$, when targeting $\mathrm{LP}_{11 \mathrm{e}}$.

When the multiplexing number is large, the converted beam includes components not from the conversion target and its degenerate modes, and so the mode converter based on phaseonly modulation is difficult to utilize in a partial MIMO-based system. 


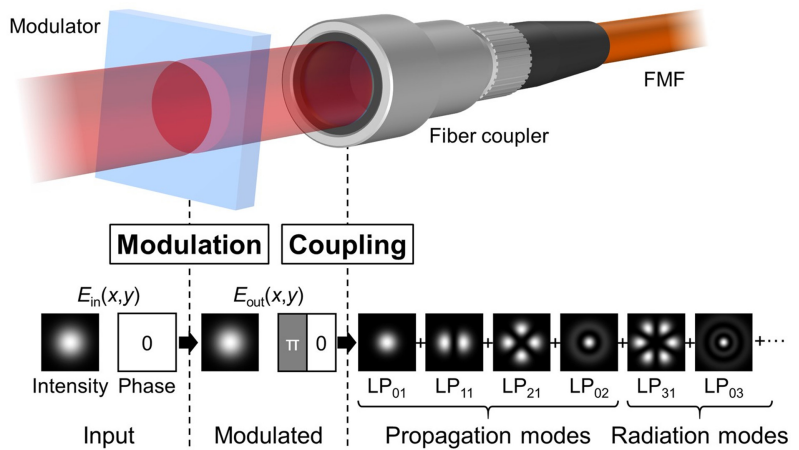

Fig. 2. Conceptual diagram of mode excitation in an FMF. The conversion method is based on phase-only modulation and the conversion target is $\mathrm{LP}_{11 \mathrm{e}}$.

\section{MODE CONVERSION BASED ON WS METHOD}

\section{A. Basic concept}

As discussed above, complex amplitude modulation that outputs only the conversion target causes significant optical loss, and phase-only modulation results in MXT. The WS method realizes a more efficient conversion than the conventional method based on complex amplitude modulation while maintaining the conversion accuracy; it does so by providing the complex amplitude modulation such that the output beam results in the WS state comprising the conversion target and the radiation modes of the FMF. Figure 3 presents images of the amplitude that is defined by the real part of the complex amplitude distribution; it also presents the cross-sectional profiles of the converted beam and its components such as "target," "crosstalk," and "others" for the three methods: phase-only, complex amplitude (conventional), and complex amplitude (WS). Here, the "crosstalk" component represents the propagation modes other than the conversion target, and the "others" component represents the radiation modes. As shown in Fig. 3(a), the beam modulated by phase-only modulation contains not only the "target" component but also the "crosstalk" and "others" components. In contrast, in the conventional method based on complex amplitude modulation, the output beam contains only the "target" component, and the output amplitude is less than the input amplitude at all positions as shown in Fig. 3(b). Although the output amplitude of the beam modulated by phase-only modulation is also less than the input amplitude, the "target" component (in the phaseonly case) partially exceeds the input amplitude. This indicates that phase-only modulation realizes a higher efficiency than conventional complex amplitude modulation by outputting the superposition state of the wavefronts of the "target" and other components ("crosstalk" and "others"). Although the "others" component is removed by the mode-filtering property of the FMF, including it in the output of the modulation can contribute to efficient mode conversion. Therefore, if the output of the WS state includes only the "target" and "others" components as shown in Fig. 3(c), efficient and accurate mode conversion can be realized.

\section{B. Derivation of the output complex amplitude distribution}

In this subsection, we explain how to obtain the WS state that includes only the "target" and "others" components. The criteria to realize efficient and accurate mode conversion are summarized as follows. (a)

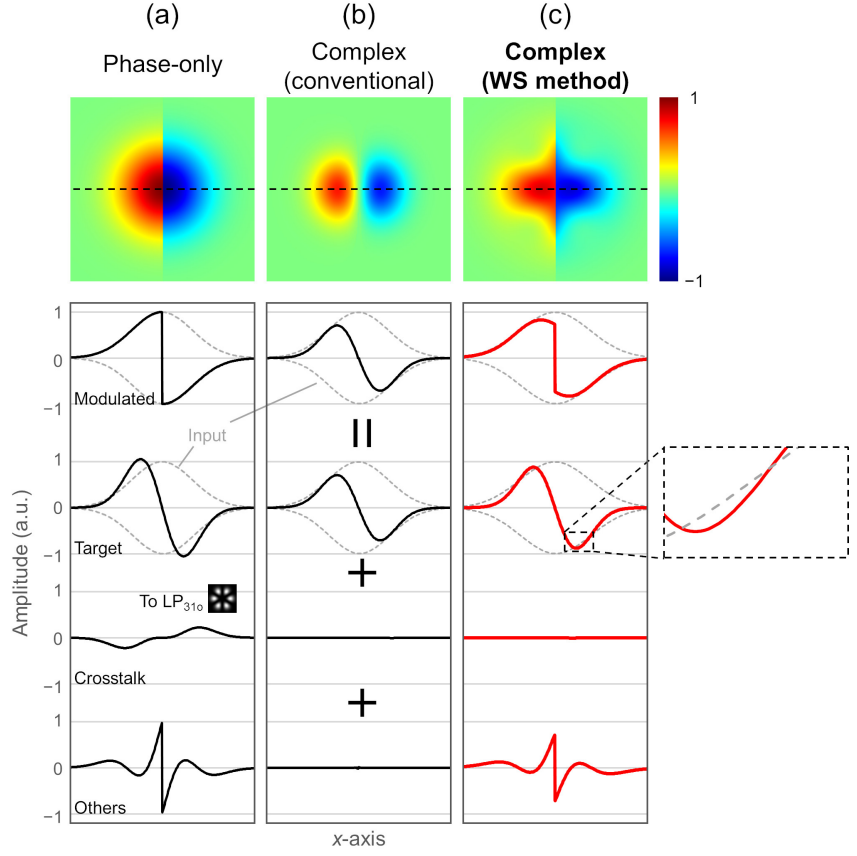

Fig. 3. Images of the real part of the complex amplitude distribution and the cross-sectional profiles of the converted beam and its components such as target, crosstalk, and others for the three methods: (a), (b), and (c). The conversion target for the 10-mode fiber is $\mathrm{LP}_{11 \mathrm{o}}$. The gray dotted lines in the modulated and target profiles represent the input amplitude. The crosstalk component in (a) mainly excites $\mathrm{LP}_{310}$. The enlarged view of the target component in (c) shows that the output by the WS method contains partially higher amplitude than the input.

- The output is composed of only the conversion target and radiation modes.

- In the target component, the output partially exceeds the input amplitude.

To satisfy these criteria, we begin from the output beam of phaseonly modulation $f_{\mathrm{p}}(x, y) \cdot E_{\mathrm{in}}(x, y)$ which is already known to contain a higher proportion of the target component. First, the coupling coefficient of the phase-only modulated beam to each propagation mode is calculated as

$$
C_{i}=\sum_{x, y}\left[f_{\mathrm{p}}(x, y) \cdot E_{\mathrm{in}}(x, y) \cdot P_{i}^{*}(x, y)\right] .
$$

Then, after subtracting the crosstalk components $C_{i} P_{i}(x, y)(i \neq \mathrm{t}$ : target) from the output, the complex amplitude is composed of only the target and radiation modes as follows:

$$
\begin{array}{r}
f_{\mathrm{p}}(x, y) \cdot E_{\mathrm{in}}(x, y)-\sum_{i=1(i \neq \mathrm{t})}^{N} C_{i} P_{i}(x, y) \\
=C_{\mathrm{t}} P_{\mathrm{t}}(x, y)+\sum_{i=N+1}^{\infty} C_{i} R_{i}(x, y) .
\end{array}
$$

By introducing a complex amplitude modulation in which the complex amplitude distribution in Eq. Eq. (6) is used instead of $E_{\mathrm{t}}(x, y)$ in Eq. Eq. (3), the components of the radiation modes are removed by the mode-filtering property of the FMF and only the component of the target mode remains to propagate in the FMF. 


\section{NUMERICAL SIMULATION OF MODE CONVERSION}

\section{A. Dependence on input beam diameter}

In the mode conversion based on phase-only modulation, it is necessary to optimize the diameter of the input beam to reduce MXT because the coupling efficiency to the propagation modes included in the modulation output depends on the input diameter. On the other hand, in the mode conversion based on complex amplitude modulation, it is possible to realize low MXT regardless of the input diameter because the output consisting of only the conversion target does not include crosstalk components. However, it is necessary to understand the input diameter dependency to maximize conversion efficiency because intensity modulation is implemented only by spatial attenuation, as mentioned in Sect. 2.A. In this subsection, we present a simulation of the coupling efficiency to the propagation modes in a 10-mode fiber while varying the input diameter in order to estimate an optimal condition for each method. The simulation was performed assuming an optical setup as shown in Fig. 4, and the simulation parameters are listed in Table 1 . In this simulation, complex amplitude modulation was implemented by providing intensity and phase modulations independently, and the propagation between the two modulators was not considered (distance between the two modulators was assumed to be zero). In addition, we introduced a reducing optical system with a magnification of $M$ between the modulator(s) and the FMF to reduce the beam diameter. This is because the pixel size of the SLM $(\sim 10 \mu \mathrm{m})$ is too large to provide the mode conversion with a sufficient resolution without the reducing optical system. Here, the reducing optical system only reduces the size of the spatial distribution of light with the magnification of the system and maintains its shape. Thus, the spatial distribution on the FMF plane can be obtained by redefining the pixel pitch of the converted light image as a reduced form with the magnification $M$. The coupling efficiency of the converted beam to each propagation mode in the FMF is

$$
\eta_{i}=\left|C_{i}\right|^{2} .
$$

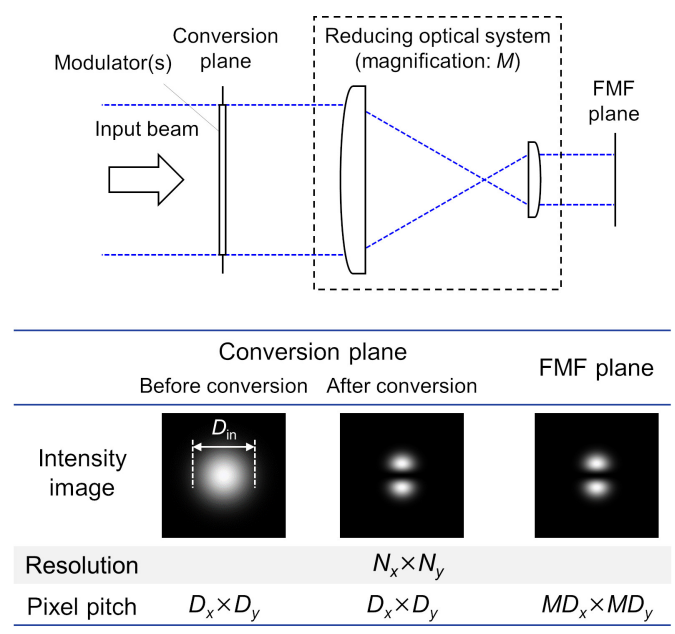

Fig. 4. Simulation model and intensity images on each plane. The spatial distribution of light on the FMF plane was obtained by redefining the pixel pitch of the image as a reduced one with the magnification of the optical system.

Table 1. Simulation parameters.

\begin{tabular}{lc}
\hline Wavelength, $\lambda(\mathrm{nm})$ & 1550 \\
Grid number, $N_{x} \times N_{y}$ & $512 \times 512$ \\
Grid interval, $D_{x} \times D_{y}\left({\left.\mu \mathrm{m}^{2}\right)}^{2}\right.$ & $10.0 \times 10.0$ \\
Modulation level of modulators & 256 \\
Magnification of reducing optical system, $M$ & $1 / 100$ \\
Input beam diameter, $D_{\text {in }}$ & Variable \\
Number of propagation modes, $N$ & 10 \\
Core diameter, $2 a(\mu \mathrm{m})$ & 15.0 \\
Normalized frequency, $V$ & 6.273 \\
\hline
\end{tabular}

Figure 5 shows the input diameter dependence for each method in the conversion targeting $\mathrm{LP}_{11 \mathrm{o}}$. In phase-only modulation [Fig. 5(a)], it is observed that not only the target mode $\left(\mathrm{LP}_{11 \mathrm{o}}\right)$ but also the crosstalk modes $\left(\mathrm{LP}_{31 \mathrm{o}}\right.$ and $\left.\mathrm{LP}_{12 \mathrm{o}}\right)$ emerge, and the MXT (defined by the ratio of the coupling efficiency to the target mode and the coupling efficiency to the other propagation modes) occurs at approximately $-10 \mathrm{~dB}$ even if the input diameter is adjusted. In the conventional complex amplitude modulation and WS methods [Figs. 5(b) and 5(c)], although the coupling efficiency to the crosstalk modes is maintained extremely low while varying the input diameter, the input diameter dependence of the coupling efficiency to the target mode is greater than that in phase-only modulation. Further, the WS method achieves higher efficiency than conventional complex amplitude modulation. In addition, the results of the conversion targeting other propagation modes and those of the other fibers, which are not shown in Fig. 5, present the same characteristics as described above. Since the MXT takes a relatively low value for all conversion targets when the coupling efficiency to the target mode is a peak value, the input diameter at this condition (summarized in Fig. 6) is defined as the optimal value and used in the following simulations.

(a)

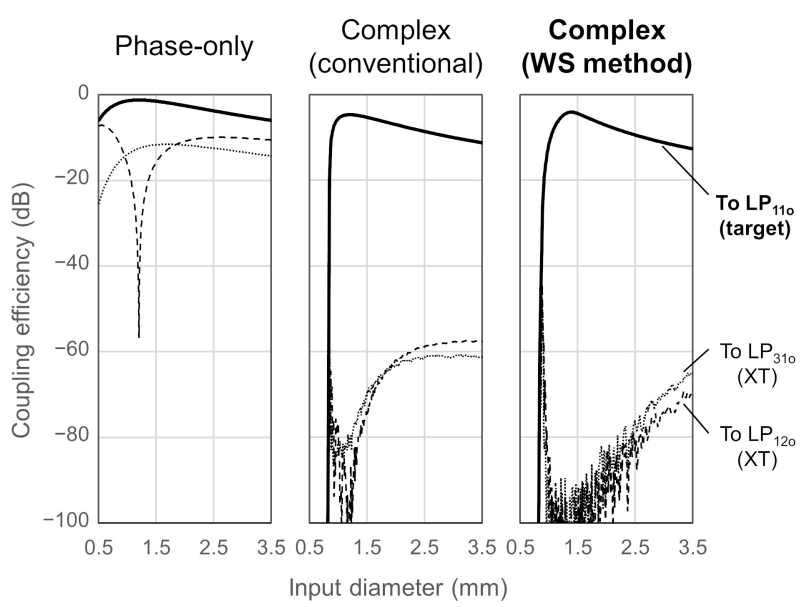

Fig. 5. Input diameter dependence for each of the methods: (a), (b), and (c). Here, values of coupling efficiency to the modes not displayed in the figure are less than $-100 \mathrm{~dB}$. The conversion target is $\mathrm{LP}_{11 \text { o }}$ of a 10-mode fiber. 


\section{B. Performance evaluation of mode conversion}

In this subsection, we numerically demonstrate that our proposed method has a higher conversion performance than the other methods even if the number of modes in the FMF increase. We examine the conversion performance of each method for FMFs supporting 3, 6, 10, and 15 propagation modes. The core diameter and normalized frequency of each fiber used in the simulation are summarized in Table 2. For simplicity, these values are estimated by changing only the core diameter from the step-index 10-mode fiber described in Ref. [28]. The input beam diameter is adjusted to the optimal value obtained in Sect. 4.A. The other parameters are the same as in Table 1. The optical loss is calculated from the coupling efficiency to the conversion target, and the MXT is calculated from the ratio of the coupling efficiency to the target mode and that to the other propagation modes as follows:

$$
X_{i}=10 \log _{10}\left(\frac{\eta_{i}}{\eta_{\mathrm{t}}}\right) \quad(i \neq \mathrm{t}),
$$

where $\eta_{\mathrm{t}}$ is the coupling efficiency to the conversion target.

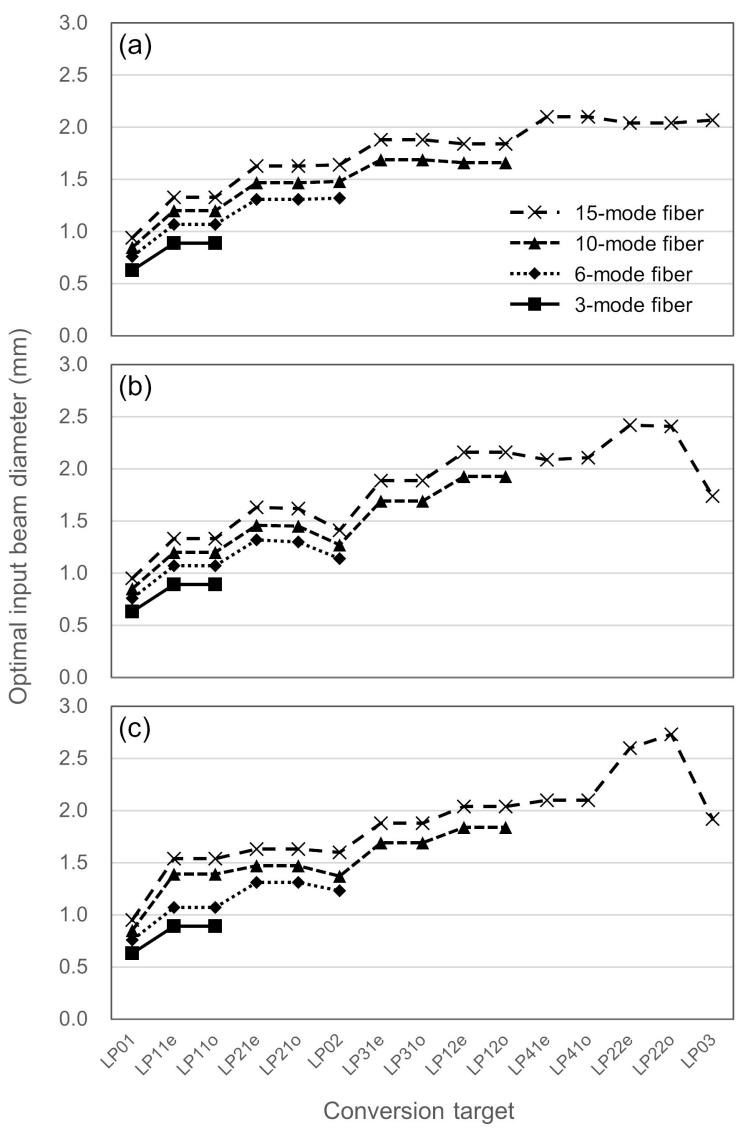

Fig. 6. Optimal input beam diameter for each fiber and each method.

Figure 7 shows the intensity distribution of the output beam targeting $\mathrm{LP}_{11 \mathrm{o}}$. As shown in Figs. $7(\mathrm{a})$ and $7(\mathrm{~b})$, the conventional methods demonstrate reasonable results that the intensity distribution of the output beam does not change significantly even if the number of propagation modes in the fiber is changed. On the other hand, in the proposed method, the output intensity distribution changes dramatically between a 6-mode fiber and a 10 -mode fiber. This is because $\mathrm{LP}_{31 \mathrm{o}}$ and $\mathrm{LP}_{12 \mathrm{o}}$ exist as
Table 2. Parameters for each FMF.

\begin{tabular}{lcccc}
\hline Number of propagation modes & 3 & 6 & 10 & 15 \\
Core diameter $(\mu \mathrm{m})$ & 8.2 & 12.0 & 15.0 & 18.5 \\
Normalized frequency & 3.432 & 5.022 & 6.273 & 7.742 \\
\hline
\end{tabular}

propagation modes in a 10-mode fiber and beyond. In a 3- or 6-mode fiber, the output beam converted by phase-only modulation targeting $\mathrm{LP}_{110}$ is orthogonal to the other propagation modes. However, in a 10- or 15-mode fiber, it is not orthogonal to $\mathrm{LP}_{31 \mathrm{o}}$ and $\mathrm{LP}_{12 \mathrm{o}}$ even if the input diameter is optimized as shown in Fig. 5. Therefore, the output distribution is changed by subtracting these crosstalk components in Eq. Eq. (6).

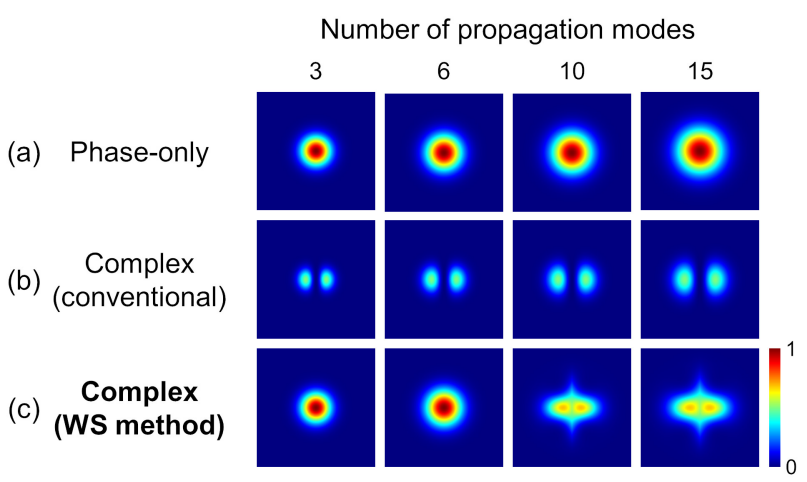

Fig. 7. Intensity distribution of the output beam modulated by each method. Each image is normalized by the peak value of input intensity. The conversion target is $\mathrm{LP}_{11}$ for each fiber.

Figures 8 (a) and 8 (b) show the maximum values of MXT and optical loss, respectively, for all conversion targets. As shown in these figures, the conversion performance decreases as the number of propagation modes increases for all the methods. However, in phase-only modulation, the maximum MXT increases remarkably, and it is $-11.3 \mathrm{~dB}$ for a 10 -mode fiber. In conventional complex amplitude modulation, although the conversion accuracy is low even for a 15-mode fiber, the optical loss is the highest for any fiber. In the WS method, although the value of optical loss jumps between a 6- and a 10-mode fiber, it maintains itself in between the values for phase-only modulation and conventional complex amplitude modulation. Moreover, in the WS method, we obtain the lowest value of maximum MXT for any fiber. It is believed that the intensity distribution after modulation is the reason why the WS method performs a more accurate conversion than conventional complex amplitude modulation. In the conventional method, large attenuation is required for the part where the intensity of the input beam is large, while the WS method requires less attenuation, i.e., the WS method can effectively use the dynamic range of intensity modulation. As a result, the WS method shows a higher efficiency of $2.4 \mathrm{~dB}$ than the conventional method based on the complex amplitude modulation, while maintaining an extremely low modal crosstalk even when the number of multiplexed modes is 15 . In conclusion, the WS method is able to realize the most accurate and relatively efficient mode conversion among the three methods. 


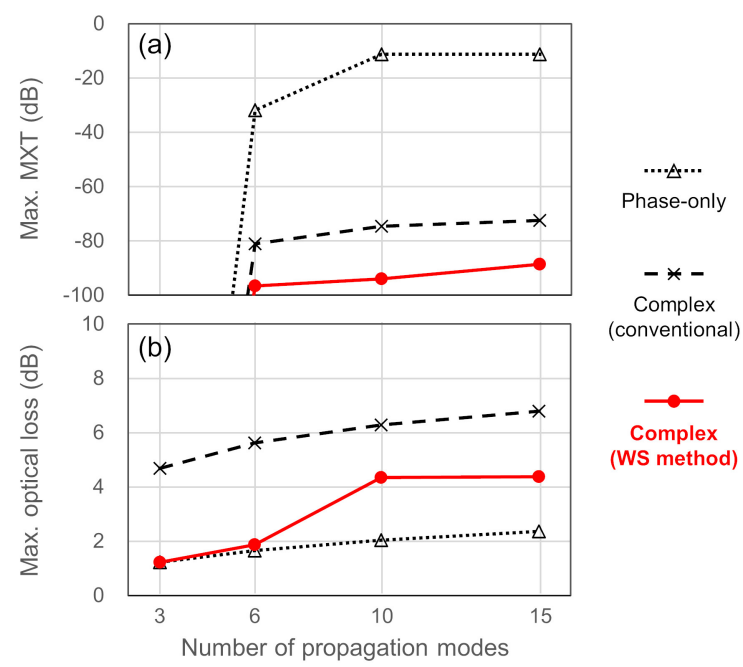

Fig. 8. Maximum value of (a) MXT and (b) optical loss occurring during conversion (for each fiber and each method). In all the methods, an MXT much lower than $-100 \mathrm{~dB}$ is obtained for the 3-mode fiber.

\section{CONCLUSION}

We proposed a wavefront superposition method using the modefiltering property of FMFs to realize accurate and efficient mode conversion. To confirm the validity of the proposed method, we numerically performed the mode conversion for FMFs supporting 3, 6, 10, and 15 propagation modes. Comparing the conversion performance with conventional methods under the optimized condition for each method, the proposed method achieved the most accurate and relatively efficient mode conversion. In this paper, the output distribution was derived starting from the output of the phase modulation. Since it cannot be said that this distribution is the best for mode conversion, in the future, it is necessary to explore a better distribution using optimization methods.

\section{REFERENCES}

1. D. J. Richardson, "Filling the Light Pipe," Science 330, 327-328 (2010).

2. R. Essiambre and R. W. Tkach, "Capacity Trends and Limits of Optical Communication Networks," Proc. IEEE 100, 1035-1055 (2012).

3. E. B. Desurvire, "Capacity Demand and Technology Challenges for Lightwave Systems in the Next Two Decades," J. Light. Technol. 24, 4697-4710 (2006).

4. Cisco Systems, Inc., "Cisco Visual Networking Index: Forecast and Trends, 2017-2022 White Paper," https://www.cisco.com/c/en/ us/solutions/collateral/service-provider/visual-networking-index-vni/ white-paper-c11-741490.html.

5. D. J. Richardson, J. M. Fini, and L. E. Nelson, "Space-division multiplexing in optical fibres," Nat. Photonics 7, 354-362 (2013).

6. G. Li, N. Bai, N. Zhao, and C. Xia, "Space-division multiplexing: the next frontier in optical communication," Adv. Opt. Photon. 6, 413-487 (2014).

7. S. Berdagué and P. Facq, "Mode division multiplexing in optical fibers," Appl. Opt. 21, 1950-1955 (1982).

8. S. Randel, R. Ryf, A. Sierra, P. J. Winzer, A. H. Gnauck, C. A. Bolle, R.J. Essiambre, D. W. Peckham, A. McCurdy, and R. Lingle, "6×56-Gb/s mode-division multiplexed transmission over $33-\mathrm{km}$ few-mode fiber enabled by $6 \times 6$ MIMO equalization," Opt. Express 19, 16697-16707 (2011).

9. R. Ryf, S. Randel, A. H. Gnauck, C. Bolle, A. Sierra, S. Mumtaz, M. Esmaeelpour, E. C. Burrows, R. Essiambre, P. J. Winzer, D. W.
Peckham, A. H. McCurdy, and R. Lingle, "Mode-Division Multiplexing Over 96 km of Few-Mode Fiber Using Coherent 6×6 MIMO Processing," J. Light. Technol. 30, 521-531 (2012).

10. K. Igarashi, D. Soma, Y. Wakayama, K. Takeshima, Y. Kawaguchi, N. Yoshikane, T. Tsuritani, I. Morita, and M. Suzuki, "Ultra-dense spatialdivision-multiplexed optical fiber transmission over 6-mode 19-core fibers," Opt. Express 24, 10213-10231 (2016).

11. D. Soma, Y. Wakayama, S. Beppu, S. Sumita, T. Tsuritani, T. Hayashi, T. Nagashima, M. Suzuki, M. Yoshida, K. Kasai, M. Nakazawa, H. Takahashi, K. Igarashi, I. Morita, and M. Suzuki, "10.16-Peta-B/s Dense SDM/WDM Transmission Over 6-Mode 19-Core Fiber Across the C+L Band," J. Light. Technol. 36, 1362-1368 (2018).

12. K. Shibahara, T. Mizuno, D. Lee, Y. Miyamoto, H. Ono, K. Nakajima, Y. Amma, K. Takenaga, and K. Saitoh, "DMD-Unmanaged Long-Haul SDM Transmission Over 2500-km 12-Core ×3-Mode MC-FMF and 6300km 3-Mode FMF Employing Intermodal Interference Canceling Technique," J. Light. Technol. 37, 138-147 (2019).

13. Y. Kokubun and M. Koshiba, "Novel multi-core fibers for mode division multiplexing: proposal and design principle," IEICE Electron. Express 6, 522-528 (2009).

14. K. Saitoh and S. Matsuo, "Multicore Fiber Technology," J. Light. Technol. 34, 55-66 (2016).

15. P. Sillard, M. Bigot-Astruc, D. Boivin, H. Maerten, and L. Provost, "Fewmode fiber for uncoupled mode-division multiplexing transmissions," in 2011 37th European Conference and Exhibition on Optical Communication, (2011), pp. 1-3.

16. P. Sillard, D. Molin, M. Bigot-Astruc, A. Amezcua-Correa, K. de Jongh, and F. Achten, "50 $\mu \mathrm{m}$ multimode fibers for mode division multiplexing," in 2015 European Conference on Optical Communication, (2015), pp. 1-3.

17. H. Zhang, W. Zhang, L. Xi, X. Tang, X. Zhang, and X. Zhang, "A new type circular photonic crystal fiber for orbital angular momentum mode transmission," IEEE Photonics Technol. Lett. 28, 1426-1429 (2016).

18. C. Brunet, P. Vaity, Y. Messaddeq, S. LaRochelle, and L. A. Rusch, "Design, fabrication and validation of an oam fiber supporting 36 states," Opt. Express 22, 26117-26127 (2014).

19. T. Hayashi, T. Nagashima, K. Yonezawa, Y. Wakayama, D. Soma, K. Igarashi, T. Tsuritani, T. Taru, and T. Sasaki, "Six-Mode 19-Core Fiber With 114 Spatial Modes for Weakly-Coupled Mode-DivisionMultiplexed Transmission," J. Light. Technol. 35, 748-754 (2017).

20. D. Soma, S. Beppu, Y. Wakayama, K. Igarashi, T. Tsuritani, I. Morita, and M. Suzuki, "257-Tbit/s Weakly Coupled 10-Mode C + L-Band WDM Transmission," J. Light. Technol. 36, 1375-1381 (2018).

21. K. Igarashi, D. Souma, T. Tsuritani, and I. Morita, "Performance evaluation of selective mode conversion based on phase plates for a 10-mode fiber," Opt. Express 22, 20881-20893 (2014).

22. W. Q. Thornburg, B. J. Corrado, and X. D. Zhu, "Selective launching of higher-order modes into an optical fiber with an optical phase shifter," Opt. Lett. 19, 454-456 (1994).

23. F. Dubois, P. Emplit, and O. Hugon, "Selective mode excitation in graded-index multimode fiber by a computer-generated optical mask," Opt. Lett. 19, 433-435 (1994).

24. S. Shwartz, M. A. Golub, and S. Ruschin, "Computer-generated holograms for fiber optical communication with spatial-division multiplexing," Appl. Opt. 56, A31-A40 (2017).

25. G. Labroille, B. Denolle, P. Jian, P. Genevaux, N. Treps, and J.-F. Morizur, "Efficient and mode selective spatial mode multiplexer based on multi-plane light conversion," Opt. Express 22, 15599-15607 (2014).

26. T. Maeda, A. Okamoto, K. Ogawa, A. Tomita, Y. Wakayama, and T. Tsuritani, "Mode conversion based on dual-phase modulation utilizing interference of two-phase-modulated beams," Opt. Rev. 25, 734742 (2018)

27. J. von Hoyningen-Huene, R. Ryf, and P. Winzer, "LCoS-based mode shaper for few-mode fiber," Opt. Express 21, 18097-18110 (2013).

28. K. Imamura, M. Yasuda, and R. Sugizaki, "Few-mode fibers with reduced mode coupling," in IEICE Tech. Rep., , vol. 114 (2014), pp. 27-30. In Japanese. 